\title{
Considerations regarding pain management and anesthesiological aspects in pediatric patients undergoing minimally invasive surgery: robotic vs laparoscopic-thoracoscopic approach
}

\author{
Francesco Molinaro ${ }^{1}$ (D) Pranvera Krasniqi ${ }^{2} \cdot$ Sabino Scolletta ${ }^{2} \cdot$ Laura Giuntini $^{2}$. Cristina Navarra ${ }^{2}$. \\ Rosa Puzzutiello ${ }^{2}$. Giulia Fusi ${ }^{1} \cdot$ Rossella Angotti $^{1} \cdot$ Edoardo Bindi $^{1}$. Clelia Zanaboni ${ }^{3} \cdot$ Mario Messina $^{1}$. \\ Girolamo Mattioli
}

Received: 4 July 2019 / Accepted: 15 July 2019 / Published online: 24 July 2019

(c) Springer-Verlag London Ltd., part of Springer Nature 2019

\begin{abstract}
In the last decade, the applicability of robotic surgery has been demonstrated in many interventions, expanding the indications of minimally invasive surgery also to pediatrics. The aim of the study is to evaluate postoperative pain to demonstrate better control following robotic procedures compared to thoraco-laparoscopic surgery. An observational, retrospective, multicentre study was performed involving 204 children undergoing robot-assisted surgery and thoraco/laparoscopic surgery at the Istituto Giannina Gaslini in Genoa and the Siena University Hospital (2013-2017): 83 children underwent robotic-assisted surgery and 121 thoracic-laparoscopic surgery. Personal data and type of intervention were assessed, dividing the patients into four categories: thoracic, gastrointestinal, hepatobiliary and urological surgeries. We analyzed the anesthetic risk according to ASA classification by type of intervention, the type of anesthesia used, the anesthetic drugs used during surgery and in the postoperative period. Both the problems that occurred during the procedures and the number of interventions converted into open during robotic surgery and laparoscopic thoracic surgery were analyzed. Pain was measured on the 1st, 2nd and 3rd day (FLACC or NRS scales). By comparing the two groups (robotics-non-robotics), the analysis shows that postoperative pain does not change with the chosen approach, but always maintains very low values, typical of minimally invasive surgery. The pain score is significantly higher in patients undergoing thoracic surgery, either robotic or thoracoscopic, compared to those undergoing gastrointestinal surgery $(P$ corrected according to Bonferroni: 0.0006$)$ and those undergoing urological intervention ( $P$ corrected according to Bonferroni: 0.04 ). In conclusion, no significant change in the intensity of postoperative pain between the two groups was found, while it is seen that the pain in patients undergoing thoracic interventions (robotic/ thoracoscopic) is more intense than that reported for other types of interventions.
\end{abstract}

Keywords Robotic surgery $\cdot$ Pediatric surgery $\cdot$ Minimally invasive surgery $\cdot$ Postoperative pain

\section{Introduction}

Francesco Molinaro

fmolidoc@me.com

1 Department of Medical Sciences, Surgery and Neuroscience, Section of Pediatric Surgery, Policlinico Le Scotte, University of Siena, Viale Bracci 14, 53100 Siena, Italy

2 Unit of Resuscitation, Critical Care, Anesthesia and Intensive Care, University Hospital of Siena, Siena, Italy

3 IRCCS Giannina Gaslini DINOGMI, University of Genova, Genoa, Italy
Robot-assisted mininvasive surgery is a useful resource in the treatment of pediatric patients. In recent years, thanks to the experience gained and the development of smaller instruments, it has been successfully applied in the pediatric field where precision is essential for carrying out reconstructive interventions [1]. Anesthesiologic management during robotic surgery includes tight monitoring of hemodynamic parameters and ventilators, careful assessment of the water balance, correct positioning of the patient at the operating table [2]. Another very important aspect in this type of surgery is pain treatment, including visceral pain due to the irritation of the peritoneum because of $\mathrm{CO}_{2}$ insufflation 
[3]. The purpose of the study was to evaluate all aspects of postoperative pain by comparing patients undergoing robotic surgery with patients undergoing the same procedure with the laparoscopic-thoracoscopic approach.

\section{Materials and methods}

We performed an observational, retrospective, multicenter study on irreversibly anonymous databases. In our study, 204 children undergoing robot-assisted surgery and thoracic/ laparoscopic surgery at the operating rooms of the Institute Giannina Gaslini of Genoa and the Azienda Ospedaliera Universitaria Senese "Santa Maria alle Scotte" were enrolled in the period from February 2015 to May 2017.

The study includes a sample of 83 children undergoing robot-assisted surgery and 121 children undergoing thoracic-laparoscopic surgery. The recovered data were analyzed, including demographic data (age of the patient on surgery, sex), classification by type of intervention; in this case, we divided our patients into four major categories (thoracic, gastrointestinal, hepatobiliary and urologic surgeries).

We analyzed the anesthesia risk according to ASA classification by type of intervention and the type of anesthesia used during surgery. We studied the most used analgesic drugs during surgery and therefore the drugs used for analgesia in the postoperative period.

Postoperative pain was evaluated by FLACC scale (Face, Legs, Activity, Cry, Consolability) in 0-7 years of age and those with psychomotor delay and Numerical Rating Scale (NRS) for elderly patients older than 7 years. Positivity of the pain assessment scale for values $>4$ was considered.

Inclusion criteria:

- Age between 0 and 15 years

- ASA I-IV

- Thoracoscopic surgery

- Laparoscopic surgery (gastrointestinal, hepatobiliary, urologic)

- Robotic-assisted thoracic surgery

- Gastrointestinal, hepatobiliary, robotic-assisted urologic surgery

Exclusion criteria:

- Age $>15$ years

- Emergency cases

Data, obtained through a review of clinical records, were collected on a paper form specially prepared for the study, and then transferred to an Excel database.
The descriptive statistics of the collected data have been performed: for the qualitative data (sex, surgical approach, etc.), the absolute frequencies and percentages have been reported; regarding quantitative variables, the median and the first and third quartiles (first to third quartile) have been reported, since data are not distributed normally. The normality of the distributions was evaluated by observing the classic descriptive measures (median, median, skewness and kurtosis) and using the Shapiro-Wilk test.

For qualitative (or categorical) data, contingency tables containing absolute frequencies and percentage frequencies have been generated; the comparison of the categorical data (frequencies) was done by the Chi square test $\left(\chi^{2}\right)$ or by the Fisher's exact test in case of expected frequencies $<5$.

Comparison of quantitative variables (such as the scale of pain) in the two groups of subjects (patients undergoing surgery in robotic surgery vs. thoracic-laparoscopic surgery) was performed by the non-parametric $U$ Mann-Whitney test. Comparison of quantitative variables (pain scale) in the four groups of subjects (patients undergoing gastrointestinal, hepatobiliary, urological or thoracic interventions) was performed by Kruskal-Wallis's non-parametric test. The use of non-parametric tests is justified by the fact that quantum data distributions did not follow a normal distribution.

Posterior comparisons after the analyses conducted on the four groups were performed by applying the Bonferroni correction to the test used for the " 2 to 2 " comparison. In this case, the significance value has been referred to as " $P$ " ".

All statistical tests were two-tier: a value of $P<0.05$ was considered statistically significant.

Microsoft Excel software was used for data management; Statistic version 9.1 (StatSoft Corp., Tulsa, OK, USA) was used for descriptive and inferential statistics analyses, while for calculating Fisher's exact test for contingency tables $3 \times 2$, $4 \times 2$ or with a number of lines $>4$, Stata version 11.0 (StataCorp LP, College Station, TX, USA) was used.

This study was validated and approved by Local Ethics Commission (number of protocol 132/2018).

\section{Results}

The study includes 204 patients, including 118 males and 86 females. The characteristics of these are shown in Table 1. As can be seen in Table 1, there is a mild prevalence of male in our population, compared to a male for a male ratio of 1.3:1. There is a wide range of age: $50 \%$ of data is in fact between 2.8 and 14.6 years. The age of patients undergoing robotic surgery (median $=7.2$ years), although slightly lower, is not significantly different from that of patients undergoing "non-robotics" (median $=10.6$ years) $(P=0.17)$. The distribution of the type of intervention is significantly different $(P<0.0001)$ in the two types of surgical approach: 
Table 1 Characteristics of the patients in the study $(N=204)$

\begin{tabular}{|c|c|c|c|}
\hline & \multicolumn{3}{|l|}{$N(\%)$} \\
\hline Sex & \multicolumn{3}{|c|}{$\begin{array}{l}\text { Male: } 118(57.8 \%) \\
\text { Female: } 86(42.2 \%)\end{array}$} \\
\hline $\begin{array}{l}\text { Age at surgery } \\
\text { (years) }\end{array}$ & $\operatorname{Median}\left(1-3^{\circ} \mathrm{q}\right)$ & \multicolumn{2}{|l|}{$9.1(2.8-14.6)$} \\
\hline Surgical approach & \multicolumn{3}{|c|}{$\begin{array}{l}\text { Robotic surgery: } 83(40.7 \%) \\
\text { Non-robotic surgery: } 121(59.3 \%)\end{array}$} \\
\hline Type of surgery & Robotic & Non-robotic & $<0.0001^{*}$ \\
\hline Thoracic & $6(7.2 \%)$ & $46(38.0 \%)$ & \\
\hline Gastrointestinal & $36(43.4 \%)$ & $38(31.4 \%)$ & \\
\hline Hepatobiliary & $5(6.0 \%)$ & $14(11.6 \%)$ & \\
\hline Urological & $36(43.4 \%)$ & $23(19.0 \%)$ & \\
\hline $\begin{array}{l}\text { Age at surgery } \\
\text { (years) }\end{array}$ & $7.2(2.7-13.3)$ & $10.6(2.9-14.9)$ & $0.17 * *$ \\
\hline
\end{tabular}

*P: Chi squared test

**P: Mann-Whitney $U$ test

urological interventions are most often conducted with a "robotic" approach, while thoracic ones are most often conducted with a "non-approach robotic ". Gastrointestinal interventions, however, are conducted both with "robotic" and "non-robotic" approaches.

Analyzing the ASA parameter by type of intervention on 196 children (i.e., patients whose parameter was possible), it was seen (Table 2) that most patients had an ASA value of $1(n=8342.3 \%)$ or $2(n=86,43.9 \%)$ (especially ASA 1 in hepatobiliary and urological interventions, ASA 2 for gastrointestinal and 43 thoracic), while ASA 3 is much less and ASA 4 values are present only in small percentages in gastrointestinal and thoracic surgery patients (Table 2 and Fig. 1).

The two anesthesiological techniques considered are balanced anesthesia and TCI anesthesia (Table 3). TCI (target controlled infusion) anesthesia is a variant of TIVA (total intravenous anesthesia) in which computerized infusional pumps, adapted to the physical characteristics of the patient (age, sex, weight, height, etc.), provide an estimate of the blood concentration of infusions anesthetized; this anesthesiologic mode is most frequently used in our study population and was homogeneously distributed between the group

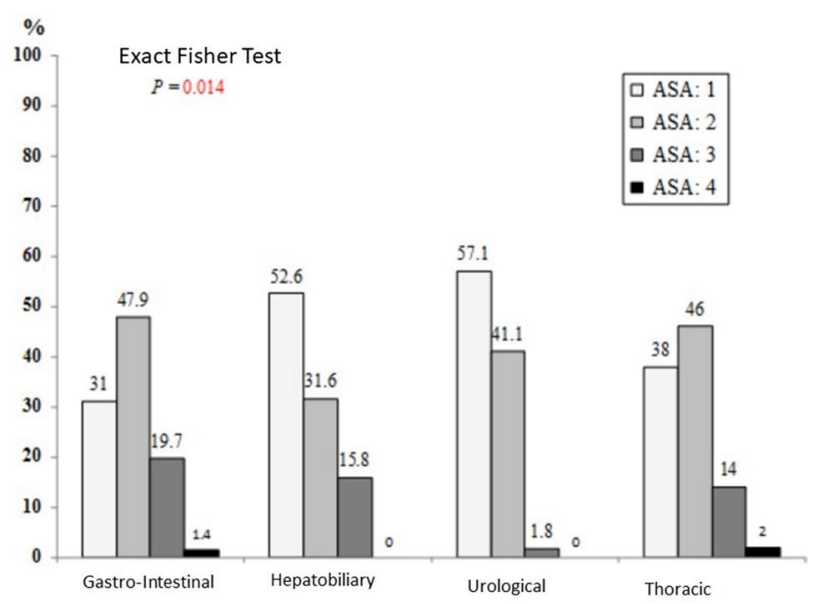

Fig. 1 ASA by type of surgery $(N=196)$

undergoing robotic surgery (TCI used in $87.1 \%$ of cases) and the group undergoing thoraco-laparoscopy (TCI in 88.5\%). Balanced anesthesia, however, involves the combination of hypnotics by inhalation and intravenous analgesics, and was only exploited in $12.9 \%$ and $11.5 \%$ of cases, respectively, robotic and thoracic-laparoscopic surgery.

The evaluation of intraoperative analgesia focused on the two most commonly used analgesics: fentanyl and remifentanil.

The results show a significant difference in the use of these drugs in the two types of surgical approach: in the thoracic-laparoscopic surgical interventions, the most used drug was fentanyl (67.3\%), while remifentanil had a much more frequent use in robotic surgery $(71.8 \%$ ) (Table 4; Fig. 2).

Regarding postoperative analgesia, the drugs under consideration were morphine (PCA), paracetamol, tramadol, ketorolac, ibuprofen, and peridural analgesia obtained with a local anesthetic (typically levobupivacaine) (Fig. 3).

The pain was measured on the 1st, 2nd and 3rd day using FLACC or NRS scales depending on the patient's age. Comparing the two groups (robotic-non-robotic), the analysis shows how postoperative pain does not change with the choice of the chosen approach (Table 5), but always remains
Table 2 ASA by type of surgery $(N=196)$

\begin{tabular}{llccr}
\hline & ASA $=1$ & ASA $=2$ & ASA $=3$ & \multicolumn{1}{c}{ ASA=4 } \\
& $N(\%)$ & $N(\%)$ & $N(\%)$ & \multicolumn{1}{c}{$N(\%)$} \\
\hline Gastrointestinal & $22 / 71(31 \%)$ & $34 / 71(47.9 \%)$ & $14 / 71(19.7 \%)$ & $1 / 71(1.4 \%)$ \\
Hepatobiliary & $10 / 19(52.6 \%)$ & $6 / 19(31.6 \%)$ & $3 / 19(15.8 \%)$ & $0 / 19(0 \%)$ \\
Urological & $32 / 56(57.1 \%)$ & $23 / 56(41.1 \%)$ & $1 / 56(1.8 \%)$ & $0 / 56(0 \%)$ \\
Thoracic & $19 / 50(38 \%)$ & $23 / 50(46 \%)$ & $7 / 50(14 \%)$ & $1 / 50(2 \%)$ \\
Total & $83(42.3 \%)$ & $86(43.9 \%)$ & $25(12.8 \%)$ & $2(1 \%)$ \\
\hline
\end{tabular}

Fisher's exact test: $P=0.014$ 
Table 3 Type of anesthesia

\begin{tabular}{lrll}
\hline & Robotic & Thoraco-laparoscopic & $P$ \\
\hline Anesthesia & & & \\
Balanced & $9 / 70(12.9 \%)$ & $12 / 104(11.5 \%)$ & $0.79 *$ \\
TCI & $61 / 70(87.1 \%)$ & $92 / 104(88.5 \%)$ & \\
\hline
\end{tabular}

*Chi squared test

Table 4 Intra- and postoperative analgesia of thoraco-laparoscopic surgery and robotic surgery

\begin{tabular}{|c|c|c|c|}
\hline & Robotic & $\begin{array}{l}\text { Thoraco-laparo- } \\
\text { scopic }\end{array}$ & $P$ \\
\hline \multicolumn{4}{|c|}{ Intraoperative analgesia } \\
\hline Fentanyl & $\begin{array}{l}\text { Yes: 35/71 (49.3\%) } \\
\text { No: 36/71 (50.7\%) }\end{array}$ & $\begin{array}{c}\text { Yes: } 70 / 104 \\
(67.3 \%) \\
\text { No: } 34 / 104 \\
(32.7 \%)\end{array}$ & 0.017 \\
\hline Remifentanil & $\begin{array}{l}\text { Yes: } 51 / 71(71.8 \%) \\
\text { No: } 20 / 71(28.2 \%)\end{array}$ & $\begin{array}{c}\text { Yes: } 49 / 104 \\
(47.1 \%) \\
\text { No: } 55 / 104 \\
(52.9 \%)\end{array}$ & 0.001 \\
\hline \multicolumn{4}{|c|}{ Postoperative analgesia } \\
\hline Morphine (PCA) & $51 / 75(68 \%)$ & $72 / 104(69.2 \%)$ & $0.86^{*}$ \\
\hline Paracetamol & $61 / 75(81.3 \%)$ & $83 / 104(79.8 \%)$ & $0.80 *$ \\
\hline Tramadol & $3 / 75(4 \%)$ & $5 / 104(4.8 \%)$ & $1.00 * *$ \\
\hline Ketorolac & $51 / 75(68 \%)$ & $75 / 104(72.1 \%)$ & $0.55 *$ \\
\hline Ibuprofen & $1 / 75(1.3 \%)$ & $3 / 104(2.9 \%)$ & $0.64 *$ \\
\hline Peridural & $8 / 75(10.7 \%)$ & $4 / 104(3.8 \%)$ & $0.07 *$ \\
\hline
\end{tabular}

*Chi squared test

**Fisher's exact test

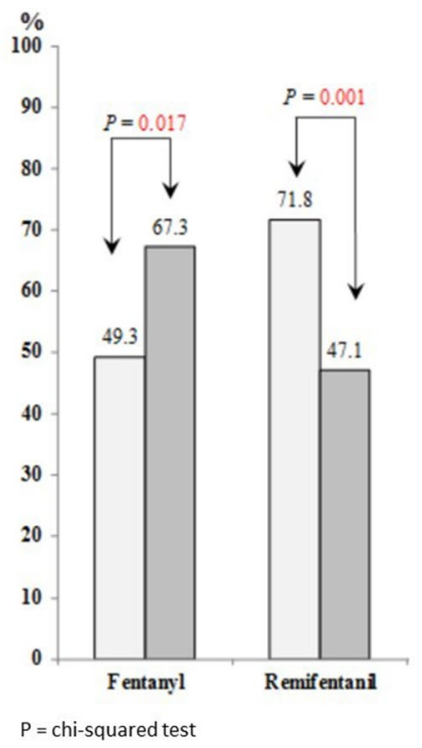

Fig. 2 Intra-operative analgesia of thoraco-laparoscopic surgery and robotic surgery at very low values (median $=0$ ), typical of mininvasive surgery.

If we stratify the entire study population (patients undergoing robotic surgery and patients undergoing thoracic-laparoscopic surgery) for the type of intervention received (gastrointestinal, hepatobiliary, urologic and thoracic), we see that there are differences in the perception of pain experienced by the different groups.

On the 1st day, the pain median always stays at 0 , for all interventions (Fig. 4).

On the 2nd day, it is noted that the score of pain is significantly higher in patients undergoing thoracic surgery than those who underwent gastrointestinal surgery (Bonferroni's correct $P$ : 0.0006) and those who underwent urological intervention ( $P$ corresponding to Bonferroni: 0.04) (Fig. 4).

The same applies to the $3 \mathrm{rd}$ day, in which thoracic postsurgical pain continues to detach itself from that of the other groups (Fig. 5).

By eliminating the group of patients undergoing thoracic surgery and by dividing the population again in patients of robotic surgery/patients of thoracic-laparoscopy, we see how the manifestations of pain are the same, both in the 1st and 2 nd and then in the 3rd day (Figs. 6, 7, 8, 9).

\section{Discussion}

Minimally invasive robotic-assisted surgery is a useful resource in the management of pediatric patients [4]. In recent years, thanks to the experience gained and the development of smaller instruments, it has been successfully applied in the pediatric field where precision is essential for the execution of reconstructive interventions [5, 6].

Anesthetic management during robotic surgery includes close monitoring of hemodynamic and ventilator parameters, careful assessment of the water balance, correct positioning of the patient at the operating table [7]. Another very important aspect in this type of surgery remains the treatment of pain, including visceral pain due to irritation of the peritoneal $\mathrm{CO}_{2}$ insufflation [8]. There are not many studies in pediatric studies regarding the anesthetic aspects and postoperative pain control [9]. Harel et al. compared open and minimally invasive surgery with respect to postoperative pain in children and they found that robotic ureteral reimplantation was associated with lower narcotic requirement compared to open surgery, and lower intensity of postoperative pain according to a direct pain assessment tool [10]. Munoz et al. studied the anesthetic aspects of pediatric robotic surgery and they stated that to provide safe and effective anesthesia for this type of surgery, it is important to have a thorough understanding of the multiple physiologic derangements that occur with robot-assisted laparoscopic surgery in infants and children, the potential complications that can occur with this 
Fig. 3 Post-operative analgesia of thoraco-laparoscopic surgery and robotic surgery

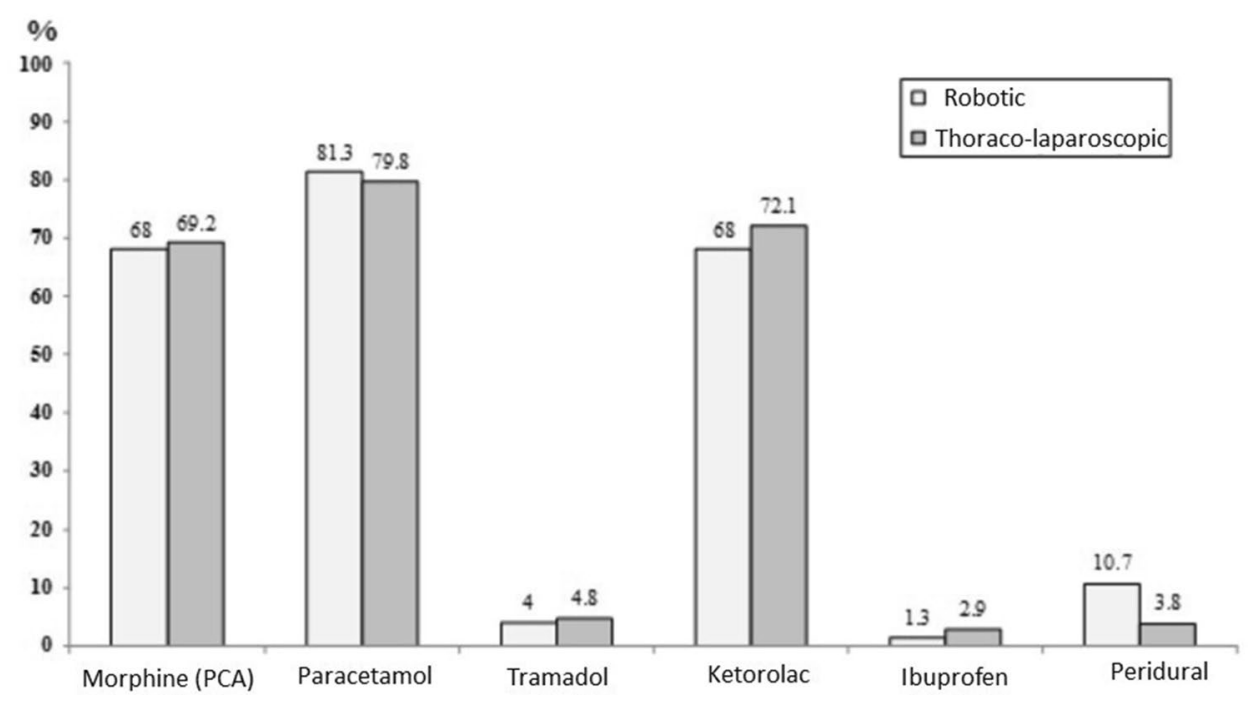

Table 5 Pain scale (FLACC/NRS) in patients undergoing robotic and thoraco-laparoscopic surgery

\begin{tabular}{|c|c|c|c|c|c|}
\hline \multicolumn{3}{|l|}{ Robotic } & \multicolumn{3}{|c|}{ Thoraco-laparoscopic } \\
\hline $\begin{array}{l}\text { Pain scale } \\
\text { FLACC/NRS }\end{array}$ & $N$ & $\begin{array}{l}\text { Median (1-3rd } \\
\text { quartile) }\end{array}$ & $N$ & $\begin{array}{l}\text { Median (1-3rd } \\
\text { quartile) }\end{array}$ & $P$ \\
\hline $1^{\circ}$ day & 55 & $0(0-2)$ & 74 & $0(0-2)$ & 0.16 \\
\hline $2^{\circ}$ day & 55 & $0(0-2)$ & 86 & $0(0-2)$ & 0.77 \\
\hline $3^{\circ}$ day & 53 & $0(0-0)$ & 84 & $0(0-2)$ & 0.11 \\
\hline \multicolumn{6}{|c|}{ Gastrointestinal surgery } \\
\hline $1^{\circ}$ day & 22 & $0(0-2)$ & 23 & $0(0-0)$ & 0.24 \\
\hline $2^{\circ}$ day & 23 & $0(0-1)$ & 25 & $0(0-0)$ & 0.25 \\
\hline $3^{\circ}$ day & 22 & $0(0-0)$ & 23 & $0(0-0)$ & 0.89 \\
\hline \multicolumn{6}{|c|}{ Hepatobiliary surgery } \\
\hline $1^{\circ}$ day & 3 & $0(0-5)$ & 12 & $0(0-4)$ & - \\
\hline $2^{\circ}$ day & 3 & $0(0-2)$ & 11 & $0(0-5)$ & - \\
\hline $3^{\circ}$ day & 3 & $0(0-0)$ & 8 & $0(0-0.5)$ & - \\
\hline \multicolumn{6}{|c|}{ Urological surgery } \\
\hline $1^{\circ}$ day & 29 & $0(0-2)$ & 21 & $0(0-0)$ & 0.06 \\
\hline $2^{\circ}$ day & 27 & $0(0-2)$ & 19 & $0(0-1)$ & 0.69 \\
\hline $3^{\circ}$ day & 25 & $0(0-0)$ & 15 & $0(0-0)$ & 0.42 \\
\hline \multicolumn{6}{|c|}{ Thoracic surgery } \\
\hline $1^{\circ}$ day & 1 & $2(2-2)$ & 18 & $0(0-2)$ & - \\
\hline $2^{\circ}$ day & 2 & $6.5(5-8)$ & 31 & $2(0-3)$ & - \\
\hline $3^{\circ}$ day & 3 & $3(0-4)$ & 38 & $1(0-3)$ & - \\
\hline
\end{tabular}

In some cases the analytical test was not performed because the number of patients in the group considered was $<4$

approach and have a consistent approach to the anesthetic management and postoperative pain control for these procedures $[11,12]$. No one compared laparoscopic and robotic approach in children regarding these aspects.

Our study population included 204 patients, of which 118 males $(57.8 \%)$ and 86 females (42.2\%); of these, 83 patients
(40.7\%) underwent a robotic surgery, while the remaining $121(59.3 \%)$ underwent thoracic-laparoscopy surgery. The median age for intervention is 9 years $(7.2$ for robotics and 10.6 for thoracic-laparoscopic). By dividing the population by the type of intervention, the type of approach changes according to the anatomical region and the location of the surgery $(P<0.0001)$; the decreasing contribution of individual groups of robotic surgery was $43.4 \%$ for urological interventions, $43.4 \%$ for gastrointestinal, $7.2 \%$ for thoracic and $6.0 \%$ for hepatobiliary surgery; as regards thoracic-laparoscopic surgery, the distribution was different: $38.0 \%$ of thoracic, $31.4 \%$ of gastrointestinal, $19.0 \%$ of urologic interventions and $11.6 \%$ of those hepatobiliary.

By evaluating ASA score by type of intervention on 196 children, the results were: for gastrointestinal interventions, $47.9 \%$ had ASA equal to 2, 31\% ASA 1, 19.7\% ASA 3 and only $1.4 \%$ ASA 4 ; in the case of hepatobiliary surgery, $52.6 \%$ of children had an ASA of 1, 31.6\% ASA 2, 15.8\% ASA 3, and no ASA 4; for urological interventions 57.1\% was ASA 1, 41.1\% ASA 2, 1.8\% ASA 3, and in this case no ASA 4; at chest level 46\% are children with ASA 2, 38\% ASA 1, 14\% ASA 3, and 2\% ASA 4.

Fisher's exact test showed a statistically significant ASA score $(P=0.014)$ in the study population (that is, the 196 children whose recovery was possible): most patients had a single ASA value $(n=83 ; 42.3 \%)$, ASA $2(n=86 ; 43.9 \%)$, while ASA 3 was less represented, and ASA 4 values only occurred in patients undergoing surgery gastrointestinal and thoracic.

With regard to anesthesiological techniques, those taken into consideration were balanced anesthesia and target controlled infusion (TCI) anesthesia. The most commonly used method in our study population was TCI anesthesia and was homogeneously distributed among the group of patients who underwent robotic surgery (TCI in $87.1 \%$ ) and the group 
Fig. 4 Pain scale (FLACC/ NRS) in patients divided by type of surgery-1st day)

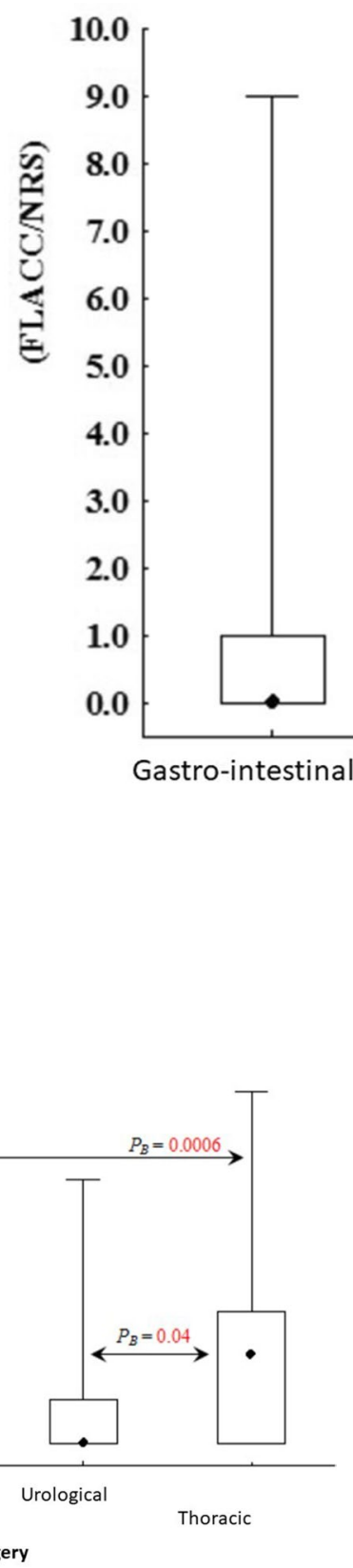

Fig. 5 Pain scale (FLACC/NRS) in patients divided by type of surgery-2nd day)

undergoing thoracic surgery-laparoscopic (TCI in 88.5\%). Balanced anesthesia was used only in $12.9 \%$ of cases of robotic surgery and in $11.5 \%$ of thoracic-laparoscopic cases.

The study, conducted on patients operated from February 2015 to May 2017, focused on postoperative pain assessment, a parameter too often underestimated by medical staff, and which, on the other hand, correlated closely with the period of hospitalization, postoperative period and outcome in general. By the population of the study at a delicate age range, postoperative pain was measured for the 3 days

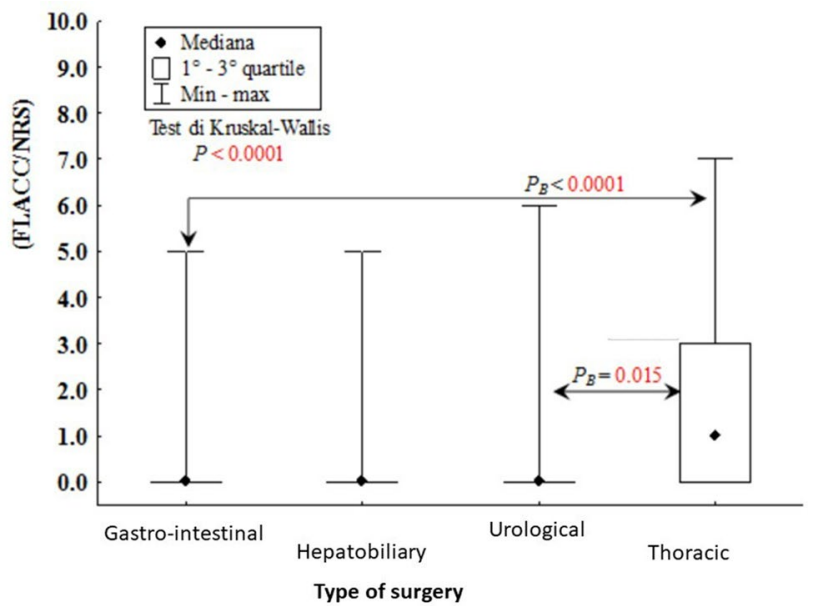

Fig. 6 Pain scale (FLACC/NRS) in patients divided by type of surgery-3rd day)

following the intervention to provide a more accurate and accurate final evidence of algae experience experienced by small patients, who they live, process and then express the painful feeling in a different and more complex way of the adult.

Analyzing the folders, we observed that intraoperative analgesic management followed two different trends, depending on the surgical approach: fentanyl opioid was considerably more used in thoracic-laparoscopic surgery, while its remifentanil derivative has had a greater use in 


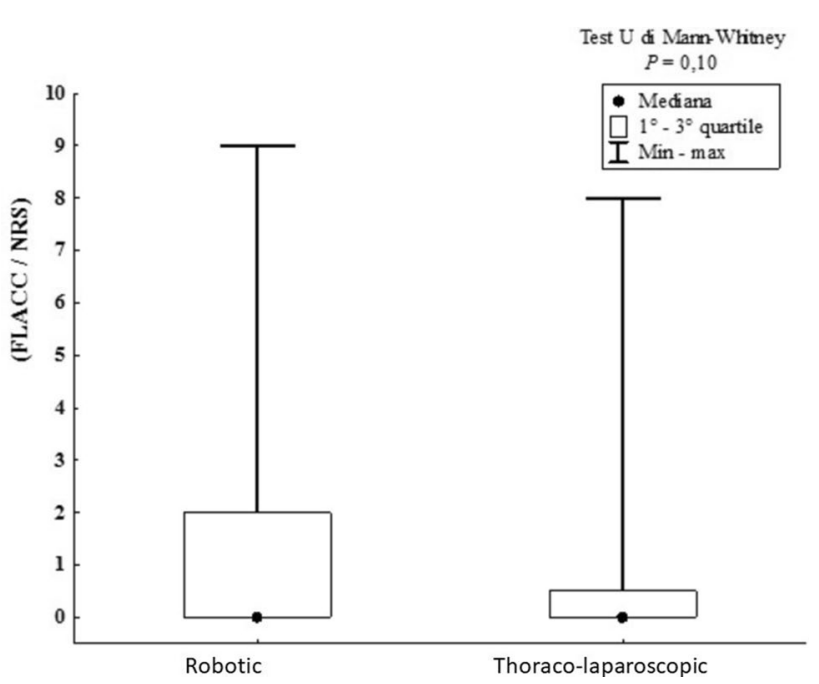

Fig. 7 Pain scale in thoraco-laparoscopic and robotic surgery (no thoracic surgery) — 1st day

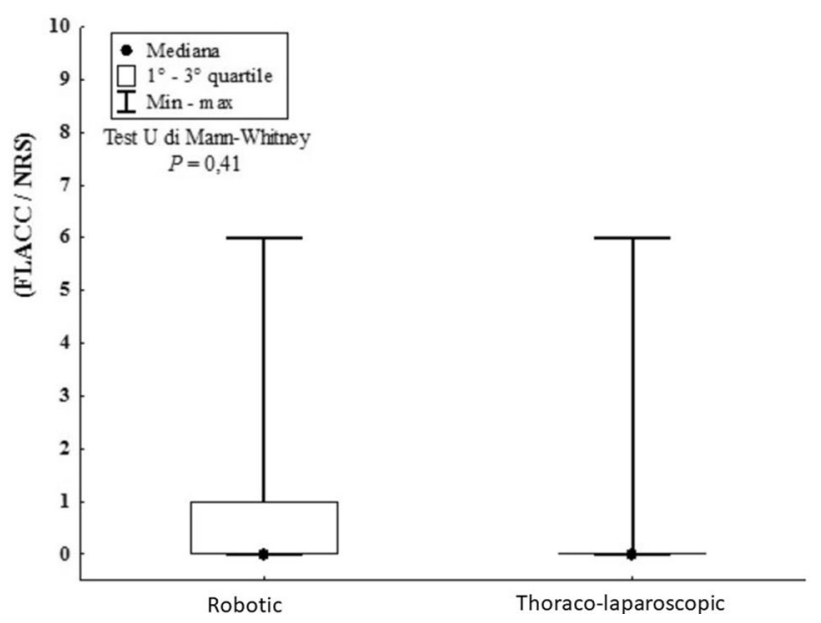

Fig. 8 Pain scale in thoraco-laparoscopic and robotic surgery (no thoracic surgery) - 2nd day

robotic surgery. Remifentanil $(P=0.001)$ was more used in robotic surgery $(71.8 \%)$, while fentanyl $(P=0.017)$ was significantly more used in thoracic-laparoscopic surgery (67.3\%).

Regarding postoperative pain management, however, statistical analyses have shown that the two comparison groups (patients undergoing surgery and robotic surgery and patients undergoing thoracic-laparoscopic surgery) did not undergo treatment with significantly different analgesic drugs, making reliable and not distorted results of our study; In fact, the majority of patients, in both groups, were treated with paracetamol, followed by ketorolac, morphine, peridural analgesia, tramadol and ibuprofen. It is noted that the above-mentioned medicines are often given in association.

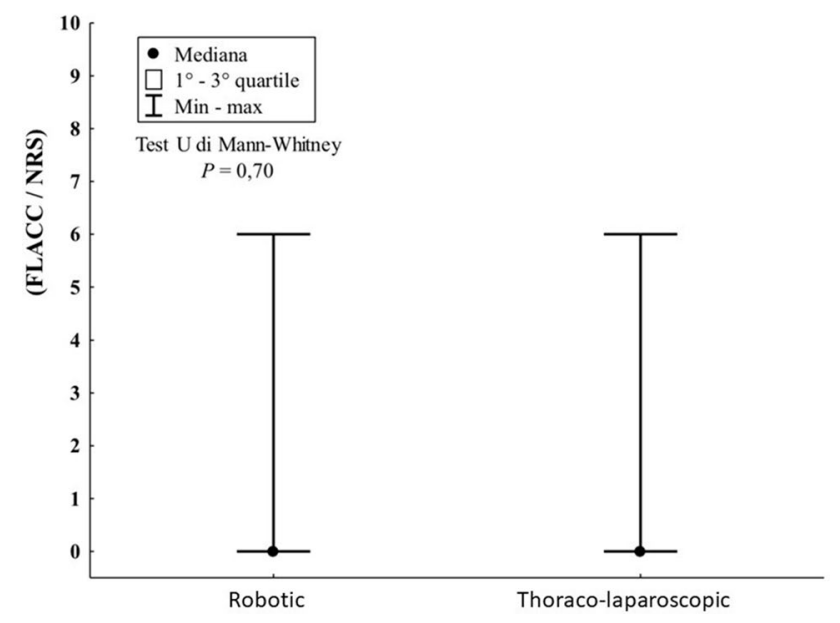

Fig. 9 Pain scale in thoraco-laparoscopic and robotic surgery (no thoracic surgery)—3rd day

Regarding postoperative analgesia, the drugs under consideration and their respective rates of use, respectively, in cases of robotic surgery and thoracic-laparoscopic surgery, were: paracetamol $(81.3 \%$ and $79.8 \%)$, morphine $(68 \%$ and $69.2 \%)$, ketorolac (68\% and $72.1 \%$ ), tramadol (4\% and $4.8 \%$ ), ibuprofen (1.3\% and $2.9 \%)$, and peridural analgesia $(10.7 \%$ and $3.8 \%)$. One important element is that the two populations (robotic and non-robotic) have been homogeneous in postoperative treatment of painkillers: this presupposes that there are no significant differences in antalgic treatment to render unsatisfactory subsequent results related to postoperative pain study, the primary goal of the research.

Postoperative pain was assessed, measured on 1st, 2nd and 3rd days using FLACC or NRS scales. By comparing the two groups (robotic-non-robotic), the results show that postoperative pain does not vary by modifying the surgical approach, but it always remains at a median value of 0 , even by comparing the two surgical approaches by type of intervention. Also in some cases (patients undergoing thoracic and hepatic biliary disorders) the statistical test was not performed, as the number of patients in the group considered was $<4$.

By stratifying the entire study population (204 patients) for the type of intervention received (gastrointestinal, hepatobiliary, urological and thoracic), and comparing the various groups by the Kruskal-Wallis test, there are differences in the experience of pain. On the 1st day, the median of pain remains at a value of 0 for all interventions. On the 2 nd day, the pain reported by small patients undergoing thoracic interventions is more intense than the children who have undergone other types of interventions; by comparing the group of chest surgery with that of urological surgery, the Mann-Whitney $U$ Test corrected by Bonferroni shows a significant difference in pain ( $P$ according to Bonferroni: 0.04$)$, 
and even more with the group of gastrointestinal surgery ( $P$ according to Bonferroni: 0.0006). The same trend is maintained on the 3rd day, where children undergoing thoracic surgery experience more pain than those under urological (Bonferroni's correct $P$ 0.015) and gastrointestinal surgeries ( $P$ according to Bonferroni: $<0.0001$ ).

The conclusion of these evidences is that children undergoing thoracic, robotic or thoracoscopic surgery, report a significantly more intense pain experience in the 2nd and 3rd postoperative days than patients in the gastrointestinal, hepatobiliary and urological levels. At this point, by eliminating the group of patients undergoing thoracic interventions (which has deviated from the others), and again dividing the population into patients undergoing robotic surgery and patients undergoing thoracic-laparoscopy, the Mann-Whitney $U$ test shows how the painful manifestations are united, whether on the 1 st and 2 nd and 3rd days.

\section{Conclusion}

The results of our research show that there was no significant modification in the intensity of postoperative pain between the two groups undergoing analysis (patients undergoing robotic surgery and patients undergoing thoracic-laparoscopic surgery), which remained sufficiently stable on low values (close to 0 in the FLACC or NRS scales) characteristic of the mininvasive surgery. Stratifying the entire population for the type of intervention received (gastrointestinal, hepatobiliary, urological and thoracic), it emerges that the pain reported by patients undergoing thoracic, robotic or thoracoscopic surgery, is significantly more intense than that reported by children who have immediately the other types of interventions (this was true on the 2nd and 3rd day). Unfortunately, the reduced number of patients undergoing thoracic surgery prevented a statistical analysis to compare the pain differences between the robot-assisted thoracic surgery group and the group of thoracoscopic surgery; Future studies comparing the above classes of patients are therefore desirable.

\section{Compliance with ethical standards}

Conflict of interest Drs Francesco Molinaro, Pranvera Krasniqi, Sabino Scolletta, Laura Giuntini, Cristina Navarra, Giulia Fusi, Chiara
Pellegrino, Rossella Angotti, Edoardo Bindi, Clelia Zanaboni, Mario Messina, Girolamo Mattioli have no conflicts of interest or financial ties to disclose.

\section{References}

1. Jackson HT, Kane TD (2014) Advanced in minimally invasive surgery in pediatric patient. Adv Pediatr 61(1):149-195

2. Cundy TP, Marcus HJ, Hughes-Hallett A, Khurana S, Darzi A (2015) Robotic surgery in children: adopt now, await, or dismiss? Pediatr Surg Int 12:1119-1125

3. Chaussy Y, Becmeur F, Lardy H, Aubert D (2013) Roboticassisted surgery: current status evaluation in abdominal and urological pediatric surgery. J Laparoendosc Adv Surg Tech 23(6):530-538

4. Boysen WR, Gundeti MS (2017) Robot-assisted laparoscopic pyeloplasty in the pediatric population: a review of technique, outcomes, complications, and special considerations in infants. Pediatr Surg Int 33:925-935

5. Bansal D, Cost NG, De Floor WR, Reddy PP, Minevich EA, Vanderbrink BA, Alam S, Sheldon CA, Noh PH (2013) Infant robotic pyeloplasty: comparison with an open cohort. J Pediatr Urol 10(2):380-385

6. Howe A, Kozel Z, Palmer L (2017) Robotic surgery in pediatric urology. Asian J Urol 1:55-67

7. Chana YY, Durbin-Johnsonb B, Sturma RM, Kurzrock EA (2017) Outcomes after pediatric open, laparoscopic, and robotic pyeloplasty at academic institutions. J Pediatr Urol 13(1):49.e1-49.e6

8. Bansal D, Defoor WR, Reddy PP, Minevich EA, Noh PH (2013) Complications of robotic surgery in pediatric urology: a single institution experience. Urology 82(4):917-920

9. Sullivan MJ, Frost EAM, Lew MW (2008) Anesthestic care of the patient for robotic surgery. Middle East J Anaesthesiol 19(5):967-982

10. Harel M, Herbst KW, Silvis R, Makari JH, Ferrer FA, Kim C (2015) Objective pain assessment after ureteral reimplantation: comparison of open versus robotic approach. J Pediatr Urol 11(2):82.e1-82.e8

11. Muñoz CJ, Nguyen HT, Houck CS (2016) Robotic surgery and anesthesia for pediatric urologic procedures. Curr Opin Anaesthesiol 29(3):337-344

12. Mattioli G, Molinaro F, Paraboschi I, Leonelli L, Mazzola C, Arrigo S, Mancardi M, Pini Prato A, Angotti R, Mario Messina M, Bianchi A (2017) Robotic-assisted minimally invasive total esophagogastric dissociation for children with severe neurodisability. J Laparoendosc Adv Surg Tech 27(5):550-555

Publisher's Note Springer Nature remains neutral with regard to jurisdictional claims in published maps and institutional affiliations. 